\title{
A Health Monitoring System based on Wearable Equipment
}

\author{
Yang LI ${ }^{1, a, *}$, Yanlian ZHANG ${ }^{2, b}$, Bangguo $\mathrm{LV}^{3, \mathrm{c}}$ \\ ${ }^{1}$ DIGITAL CHINA(CHINA)LIMITED, Beijing, China \\ ${ }^{2}$ China Flight test establishment, Xi’an,China \\ ${ }^{3}$ Jilin JLU Communication Design Institute Co., Ltd. Changchun, China \\ a digital9898@sina.com
}

Keywords: Wearable Equipment; Remote Health; Fall Detection

\begin{abstract}
Monitoring the health of the elderly people through wearable equipment helps to reduce the health and medical expenses associated with falls in the elderly people. The technology of falling detection is analyzed, and a fall detection and alarm system based on triaxle acceleration sensor and gyroscope is proposed. Three-axis acceleration sensor and gyroscope real-time collect data from the elderly people in the daily activities of the acceleration and angular velocity data. The software running on the mobile phone to analyze the data received and determines the movement of the elderly people state. When the system detects a fall occurs, the ringing tone to remind the elderly, the elderly people can confirm the alarm or cancel false positives. If there is no response within 20s, the system will notify the contact according to the set alarm by means of messages and call. The elderly can also alarm when feel ill.
\end{abstract}

\section{Introduction}

In recent years, wearable equipment and telemedicine has become a hot topic of research. With a wide variety of wearable equipment appeared, the IT industry and the media pay more attention on this area. Healthy bracelets as the representative of the medical health wearable equipment is one of the most sought. People's demand for personal health care has been existed, and with the development of embedded technology, cloud computing technology and sensor technology in recent years, the development of services to individuals in the small wearable equipment environment gradually mature [1] [2].

Wearable health monitoring technology is an effective way to achieve combination of hospitals, communities, families and individuals. It is an effective way to alleviate people's need for health monitoring and limited medical resources. Wearable health monitoring system has become one of the emerging international hotspots in today's international concern. Based on the wearable fall detection system, a lot of research had been done in domestic and abroad [3] [4] [5], basically through a variety of sensors to collect human activity data, and through a certain algorithm to determine whether the elderly people fall. And take the appropriate measures to play a role in health status and location confirmation.

\section{Key Technology}

The fall is caused by the fact that the body lost the balance, and in the event of falling itself can not control the dumping action. Fall is an unpredictable event that generally occurs in a short time. When the fall occurs, the body will dump in a certain moment, resulting in the body center of gravity to move down, the body fast action makes the direction of acceleration value changes.

When the fall occurs, the body position of the body from the standing or sitting state turned to lying state, in the process of transformation, the body parts of the displacement, speed and acceleration of the three vectors have changed. The use of a single-axis acceleration sensor and a two-axis sensor to form a sensor with three dimensions to obtain human space attitude information, and the monitor sensor is at the waist. 
In the fall detection system, the sensor is mainly used to perceive the activities of the elderly people. There are a wide variety of sensors, according to the use of principles have a specific classification. Such as resistance / capacitive sensors, temperature / pressure sensors, speed sensors, and so on. Due to the wearable needs, the sensor size should be as small as possible, so we uses MEMS sensor technology.

\section{Design of the System}

Based on the embedded development technology and the design of wearable equipment, we designs a wear device that integrates a three-axis acceleration sensor, a gyroscope, a Bluetooth 4.0 module and an Atmega 168 microcontroller module into a wearable Vest, which can collect and record the daily life of the elderly people in real-time, and all the data transmitted to the smart phone through Bluetooth, mobile phone process and analyze the data. When the detection of fall occurs, it can remind the associated phone, alarm and so on.

The architecture of the system is shown in Figure 1, sensor and Bluetooth, Atmega168 microcontrollers integrated in the user's wearable vest, used to capture and transfer to the client side of the required mobile phone activity data, smart phone software based on the fall monitoring algorithm real-time processing of the data. If a fall was detected, it can send a message and dial the phone the mobile network, the default contact person can be a guardian or a medical institution.

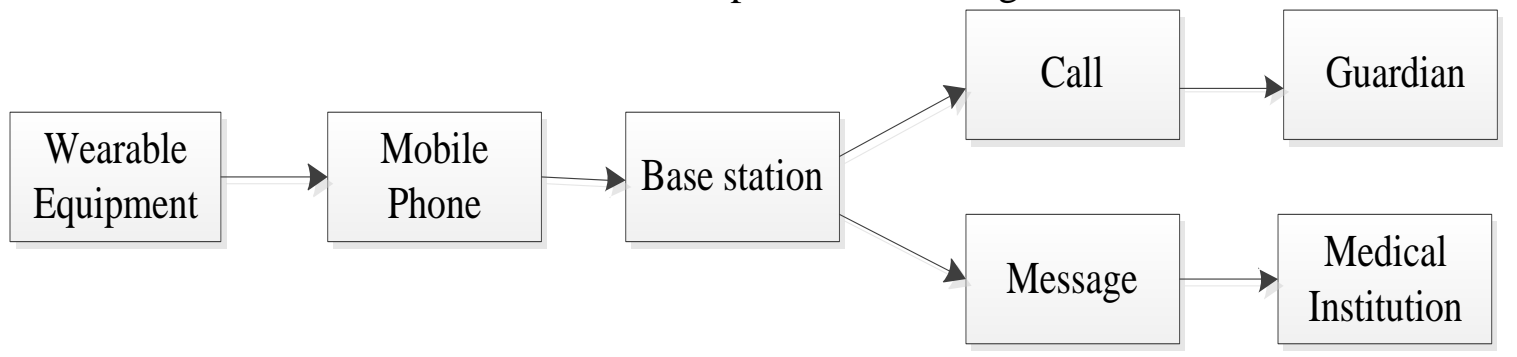

Figure1 system architecture

The hardware architecture of the system is divided into a wearable part of the device and the user's smartphone part, which can be worn by the device for self-assembly, and the smartphone is an existing tool that calls some of its modules.

In the wearable device section, the triaxle acceleration sensor and the gyroscope pass the collected data to the microprocessor, and the microprocessor transfers the collected data to the smartphone via the Bluetooth module

When Bluetooth module receives the data, the core processor data processing in real time, when the user falls, the smart phone calls GPRS module quickly to access the user's local coordinates, the software by calling map open source API to obtain the user's Map coordinates and text form of the location information, map coordinates through the mobile network communication feedback to the guardian software side, the text form of the location information through the message sent to the guardian. At the same time supports the user to call the guardian phone.

System software architecture is divided into wearing device software and smart phone software parts. Smart phones can develop part of API development, call the corresponding function modules. The data processing algorithm is the core algorithm of the design, the alarm module software design focus on the UI and interactive style of the original design, the underlying function calls android open source for the function module.

\section{Flow of Fall Monitoring}

The flow of fall monitoring is as follows:

(1) In normal circumstances, the body's acceleration value will not exceed $3 g$. When fall occurs the acceleration value can reach $6 g$, if the acceleration value exceeds the threshold $6 g$, enter the second step.

(2) When the continuous jump, the instantaneous acceleration may also be large, follow a 
relatively short period of time will still appear a large acceleration value. $2 \mathrm{~s}$ after falling, the human body in the state of the need to adjust the fall to stimulate, basically in a static state. Based on this feature, the acceleration value is detected for two seconds after the instantaneous acceleration is greater than 6g. If there is no significant change in the acceleration value, the third step is entered.

(3) Calculate the peak before the first 1 second and after 2 seconds in the tilt angle, compared to the two angles of the change, if the difference between the two angles is greater than 90 degrees, and then determine the fall occurred.

\section{Realization of Algorithm}

The main flow of the fall algorithm is shown in Figure2. The system reads the activity data within 2s. If the threshold is bigger, it will continue to compare the angular velocity. Otherwise, it will return to the previous step to read the data. Angular velocity data also exceeded the angular velocity threshold is determined to fall to alarm, otherwise return to the first step.

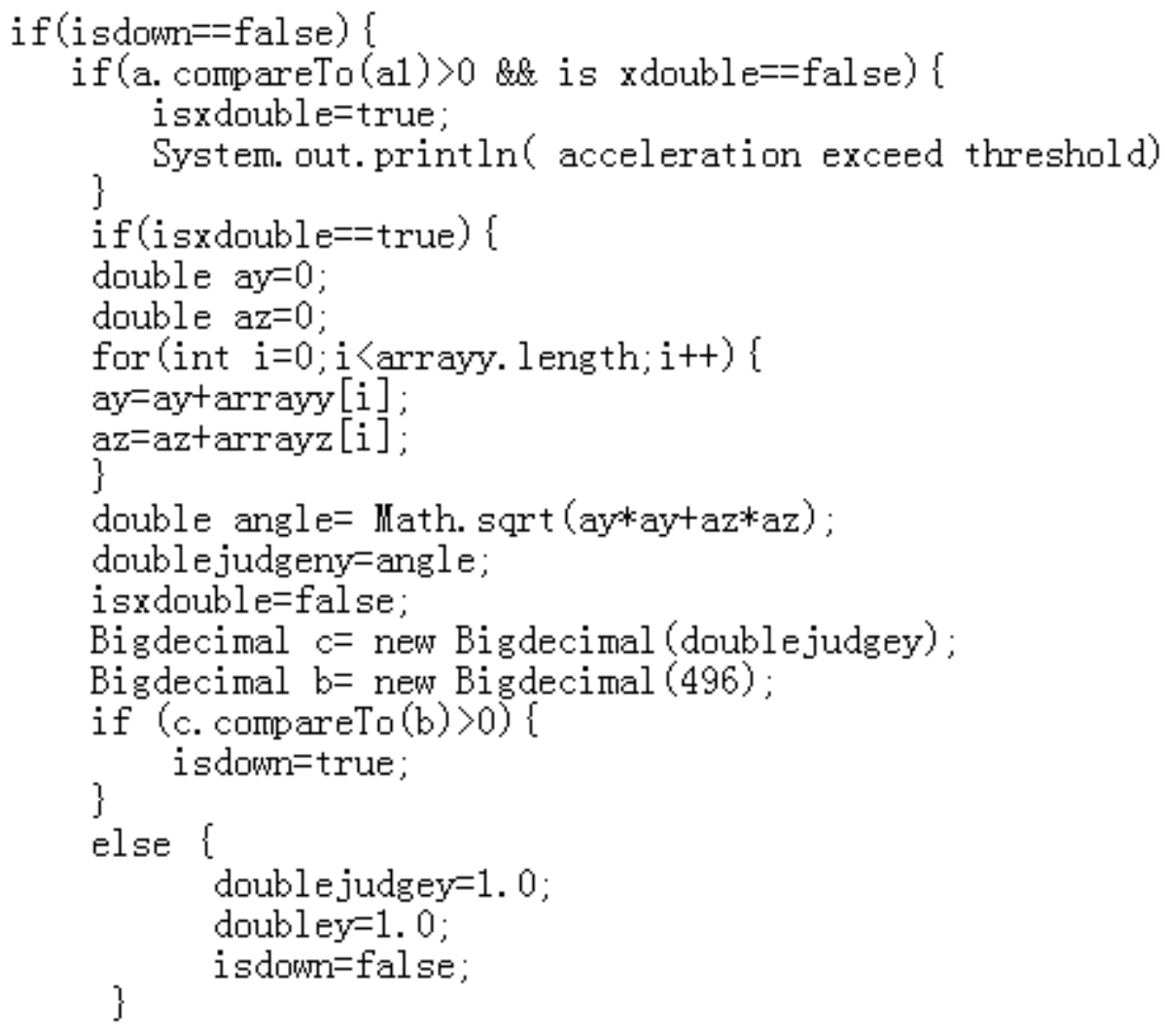

Figure2 realization of the fall algorithm

IsDown is the fall flag, because if the user is already down the state system will no longer be detected. A1 and b are the human body acceleration and the upper torso from the vertical direction of the tilt angle. When the system determines that the acceleration exceeds the threshold value will be angular velocity judgments, if the tilt also exceeds the threshold, so that isDown $=$ true and call the corresponding alarm module; if the tilt does not exceed the threshold, it may be due to some of the acceleration of the movement caused by excessive acceleration threshold, make isDown $=$ false.

\section{Conclusions}

A fall detection and alarm system based on triaxle acceleration sensor and gyroscope is provided. It aims to detect the occurrence of falls and timely alarm by means of scientific means so as to reduce the health injury and medical expenses brought by the fall of the elderly people. The overall scheme of the fall monitoring and alarm system is designed, including the system requirements and the overall architecture of the system. 


\section{References}

[1] Arman Anzanpour; Iman Azimi; Maximilian. (2017) Self-awareness in remote health monitoring systems using wearable electronics. Design, Automation \& Test in Europe Conference \& Exhibition (DATE), 1056 - 1061.

[2] Vaibhavi Bhelkar; D. K Shedge. Different types of wearable sensors and health monitoring systems: A survey. 2016 2nd International Conference on Applied and Theoretical Computing and Communication Technology, 2016, 43 - 48.

[3]Huiyu Jia; Meihui Li; Yunkun Ning. Implementation of Android-based fall-detecting system. 2016 IEEE 13th International Conference on Signal Processing (ICSP), 2016, 1323 - 1328.

[4]Matthew Chin Heng Chua. Design and development of an integrated health (i-health) monitoring watch, 2016 IEEE/SICE International Symposium on System Integration (SII), 2016, 296 - 300.

[5]Monali V Madavi; Shubhangi D Giripunje. Design and Implementation of Wearable Device for Neuropathic Diabetic Foot Patients. 2015 7th International Conference on Emerging Trends in Engineering \& Technology (ICETET), 2015, 23 - 27.

[6]Kishori C. Rajwade; Dhanashri H. Gawali. Wearable Sensors Based Pilgrim Tracking and Health Monitoring system, 2016, 1- 5. 